\title{
Tsafon
}

Revue d'études juives du Nord

$82 \mid 2021$

Enjeux esthétiques dans la littérature après Auschwitz

\section{Kolmar Gertrud, Féminité et judéité. Poèmes 1927-1937, choisis, traduits et commentés par Mireille Tabah}

Andrée Lerousseau

\section{(2) OpenEdition}

Édition électronique

URL : https://journals.openedition.org/tsafon/4462

DOI : $10.4000 /$ tsafon.4462

ISSN : 2609-6420

Éditeur

Association Jean-Marie Delmaire

Édition imprimée

Date de publication : 1 décembre 2021

Pagination : 141-143

ISSN : 1149-6630

\section{Référence électronique}

Andrée Lerousseau, « Kolmar Gertrud, Féminité et judéité. Poèmes 1927-1937, choisis, traduits et commentés par Mireille Tabah », Tsafon [En ligne], 82 | 2021, mis en ligne le 01 décembre 2021, consulté le 12 février 2022. URL : http://journals.openedition.org/tsafon/4462 ; DOI : https://doi.org/ $10.4000 /$ tsafon.4462 


\section{À travers les livres ......}

Kolmar Gertrud, Féminité et judéité. Poèmes 1927-1937, choisis, traduits et commentés par Mireille Tabah, Paris, L'Harmattan (coll. Poètes des cinq continents), 2021, 221 p. $20,50 €$.

Gertrud Kolmar, pseudonyme de Gertrud Käthe Chodziesner, cousine de Walter Benjamin, est née à Berlin en 1894 dans une famille de la bourgeoisie juive assimilée. Femme cultivée, dotée d'un don particulier pour les langues, elle se voulait libre et ouverte au monde et fut très tôt confrontée aux normes de la société conservatrice et patriarcale. Juive, qui fit le choix de demeurer auprès de son père en Allemagne, elle fut victime des persécutions dans un pays gangrené par l'antisémitisme. Contrainte au lendemain des pogromes de novembre 1938 d'emménager avec son père dans un immeuble " réservé aux Juifs », elle avoue dans une lettre à sa sœur émigrée en Suisse avoir l'impression d'errer sans fin, comme dans un mauvais rêve dont elle ne parviendrait pas à s'éveiller ${ }^{1}$. Soumise au travail forcé à partir de juillet 1941, elle sera arrêtée le 27 février 1943 durant la soi-disant Fabrikaktion ("Action dans les usines ») et déportée le 2 mars avec le «32ème convoi vers l'Est » en direction d'Auschwitz, date à laquelle un document de l'état civil allemand fixe la date de sa mort. On ignore si elle est décédée lors du transfert ou si elle fut assassinée dès son arrivée au camp d'extermination. Conséquence de l'interdiction de publication qui frappait les Juifs, mais également du peu de cas que Kolmar faisait de la notoriété, son œuvre fut publiée en Allemagne pour l'essentiel à titre posthume. En France, à l'exception des drames, elle a fait l'objet de parutions tardives, mais assez régulières si l'on considère les rééditions au format de poche ${ }^{2}$.

Les éditions de l'Harmattan proposent en version bilingue un choix de 45 poèmes, traduits et commentés par Mireille Tabah. Rédigés entre 1927 et 1937, les textes appartiennent à la seconde phase de création et peuvent se lire comme la déclinaison d'une double identité (féminine et juive), leur agencement, en particulier au début de la troisième section, soulignant le caractère indissociable des deux pans constitutifs de l'identité et le sentiment d'étrangéité, lié à la fois à la féminité et à la judéité. Mireille

\footnotetext{
${ }^{1}$ Gertrud Kolmar, Briefe. Éd. Johanna Woltmann, Göttingen : Wallstein-Verlag, 1997. Voir la lettre à sa sour Hilde, datée du 2 juin 1941.

${ }^{2}$ Cf. la liste des publications, p. 219 du présent ouvrage, à laquelle il convient d'ajouter la publication aux éditions Circé des recueils Portrait de femmes et Rêves de bêtes, rassemblés par Fernand Cambon sous le titre éminemment révélateur Quand je l'aurai tout bu (2014), et de Mon Enfant (2015) et Robespierre, et Le portrait de Robespierre (2017) dans une traduction de Sibylle Muller.
} 
Tabah, et ce n'est pas là le moindre mérite de son travail, restitue de façon exemplaire le rythme qui habite la poésie de Kolmar, un même souffle, une même respiration traversant à la fois l'original et la traduction, et elle rend au plus près le « langage poétique d'une extrême intensité, fondé sur la densité et la métamorphose constante des métaphores » (cf. sa préface), caractéristique de cette seconde période où s'ébauche l'exploration des univers intérieurs, somptueusement décrits dans le recueil Mondes (1937), et où s'affirme déjà une maîtrise souveraine de la langue qui culminera dans la parole luxuriante, la débauche langagière suscitant l'admiration par son déploiement de beauté qui distingue le dernier recueil rédigé en allemand ${ }^{3}$. Le fait que Mireille Tabah ait précisément exclu de son choix les poèmes de Mondes, qui ont tout aussi bien partie liée avec la quête et l'affirmation d'une double, voire triple identité (femme, juive et poète), permet de souligner l'importance de « cette phase d'écriture extrêmement productive » (p. 189) et d'éclairer avec rigueur le chemin emprunté par Kolmar qui, par le biais d'une « imagerie » qui lui est propre et d'un jeu subtil de métaphores, conduira en 1937 à une apothéose de la langue. Et peut-être faut-il également voir dans ce renoncement l'expression d'une reconnaissance du travail effectué par Jacques Lajarrige, traducteur de Mondes, qui le premier restitua en allemand et en français l'ordre des textes voulu par Kolmar, permettant ainsi au lecteur d'avoir une juste appréhension de cette œuvre majeure ${ }^{4}$.

Les poèmes retenus par Mireille Tabah sont répartis en trois sections. La première, intitulée «Visages de femmes », se présente comme une suite de variations autour d'un « je » lyrique qui, à travers ses figures multiples et ses incessantes métamorphoses, revendique et affirme son désir, dans une tension permanente en direction d'un « tu » masculin qui toujours se dérobe, une fois sa soif assouvie. Ainsi qu'il est mentionné dans la préface, les poèmes sont autant de voyages « hors des sentiers bourgeois », sont le creuset où se rassemblent des femmes déchues et démonisées pour avoir assumé leur sexualité ou parce que la vieillesse et l'approche de la mort dont l'ombre plane sur les derniers textes leur ont ôté toute «qualité » d'objet sexuel. Dans un partage de leur douleur et de leur solitude et par la grâce de la poésie, Kolmar leur rend leur dignité et les pare d'une beauté due à leur inquiétante et dérangeante étrangeté.

Dans le prolongement des souffrances liées à la féminité, le « Kaddish pour l'enfant qui n'a pu naître » qui constitue la seconde section, aborde le thème d'une maternité frappée d'interdiction dans une société bourgeoise et patriarcale reposant sur une double morale. Réceptacle du traumatisme vécu par Kolmar elle-même, contrainte durant sa jeunesse à un avortement, le poème devient le lieu des épiphanies et des métamorphoses de l'enfant non-né que les forces conjuguées de l'amour (confinant parfois à la folie) et de la parole font advenir à la présence. C'est cet avènement d'un être dont l'essence n'est faite que de poésie que décrit «Une autre», poème inaugural magnifiquement traduit, tout en délicatesse et d'une bouleversante beauté, où touche par touche, comme sur une toile, celle qui écrit s'imprègne de cette présence en elle afin de lui donner forme.

La dernière section, « Nous, Juifs », emprunte son titre au poème éponyme qui résonne comme une déclaration d'amour de la poétesse à son peuple : « Seule la nuit écoute : je t'aime, je t'aime, mon peuple,/ Et veux t'étreindre tout entier de mes bras brûlants et fermes » (p. 163). La plupart des textes sont extraits du recueil, inédit en français, $L a$ parole des muets (Das Wort der Stummen, que l'on peut tout aussi bien traduire, ainsi que le remarque Mireille Tabah, par La parole de la muette, car Kolmar « sait que son cri ne sera pas entendu »). Rédigé entre le 18 août et le 25 octobre 1933, au moment où

\footnotetext{
${ }^{3}$ Les ultimes poèmes, écrits en hébreu dont Kolmar fait l'apprentissage en 1941, ont été perdus.

${ }^{4}$ Gertrud Kolmar, Mondes, édition établie, postfacée et traduite par Jacques Lajarrige, Paris, Seghers, 2001.
} 
s'entamait le procès de l'incendie du Reichstag et où la Gleichschaltung (la mise au pas de toute la société allemande) battait son plein, le recueil ne fut publié qu'en 1978 en RDA, grâce aux soins de Hilde Benjamin, Ministre de la Justice de RDA de 1953 à 1967, à laquelle Kolmar avait remis le manuscrit peu de temps après la mort de son père déporté à Theresienstadt. Un événement touchant de près la poétesse a sans nul doute contribué au jaillissement inhabituel de la parole politique : la mise en détention préventive, le 12 avril 1933, de son cousin Georg Benjamin, militant communiste, suivie de son transfert au camp de concentration de Sonnenburg. Faisant preuve d'une lucidité et d'une clairvoyance dont nombre de Juifs allemands, se berçant encore de l'illusion que le national-socialisme ne serait qu'une brève parenthèse, étaient dépourvus, Kolmar fait une fois de plus cause commune avec ceux qui sont frappés d'exclusion: les prisonniers politiques d'une part, réduits à l'état de bétail promis à la mort, dont elle décrit les effroyables conditions de détention, et les Juifs, éternellement confrontés au mépris, aux persécutions et aux discriminations, stigmatisés sans relâche par le regard que pose sur eux une société marquée par le refus de toute altérité. Face à la faillite de l'amour et de la prétendue religion d'amour, Kolmar revendique haut et fort son appartenance à son peuple, dont elle se fait l'héritière, réaffirmant en son nom le rejet de toute idolâtrie et le rêve messianique juif de l'avènement d'une ère de justice (p. 165).

"L'étrangère », ignorant si son message sera entendu, accuse et s'insurge contre un univers de violence et de haine dans lequel ce qui témoigne de l'humain, le visage, est défiguré, "profané », au même titre que le corps de la femme. Avec pour armes ses mots et, ainsi qu'il est rappelé dans la préface, l'amor fati, expression de la dignité inaliénable et de la souveraineté qu'elle partage avec la femme libre, qui refuse d'être « une docile poule de ferme/ Qui pond son œuf et crételle» (p. 29), ou bafouée, et avec son peuple, elle proclame à la face du monde et des bourreaux la beauté paradoxale et l'élection des exclus : « Si pour toi je ne suis que vermine répugnante :/ Je suis le crapaud/ Et porte la pierre précieuse ...» (p. 143).

Le parti-pris de la traductrice de limiter les notes et commentaires aux seuls passages nécessitant une explication historique (cela vaut principalement pour la troisième section) ou philologique, nous renvoie au premier poème et témoigne d'une exigence que Mireille Tabah partage avec « la poétesse » (titre du poème), qui d'emblée entend placer le lecteur devant sa responsabilité : « [...] Toi qui lis ceci, fais-y attention;/ Car vois, tu feuillettes un être vivant. »; « Tu entends ce qui parle. Perçois-tu aussi ce qui ressent ? » (p. 19 et p. 21). Injonction et interrogation qui «obligent» le destinataire : à lui, en effet, de se mettre à son tour à l'écoute d'une écrivaine inclassable qui toujours se dérobe au gré des métaphores et des métamorphoses, de faire l'effort de pénétrer une œuvre unique, infiniment dense, sans la violenter.

Andrée Lerousseau

Rachel, Sur les rives de Tibériade. Traduit de l'hébreu et présenté par Bernard Grasset. Paris-Orbey, Arfuyen, 2021, 194 p. $17 €$.

Le poète et traducteur Bernard Grasset (né en 1958) revient dans cet ouvrage pour recueillir et traduire divers textes de Rachel, qui n'ont pas encore été traduits en français et n'ont pas été publiés sous forme d'un recueil de textes de Rachel en hébreu. Rachel Blaustein (1890-1931), connue sous son prénom «Rachel», est l'une des poétesses fondatrices les plus appréciées de l'histoire de la littérature hébraïque moderne. Trois livres de poésie en hébreu recueillent ses poèmes les plus connus, dont deux ont été publiés au cours des cinq dernières années de sa courte vie : Saphiah (Regain), 1927 et Minégéd (De loin), 1930 ; et le dernier qui a été publié après sa mort : Nébo, 1932. Les poèmes de ces trois livres ont été préalablement traduits en français par Bernard Grasset, 
et ont été publiés dans une version bilingue, également par les Éditions Arfuyen : Regain, en 2006 ; et De loin suivi de Nébo, en 2013.

Ce volume inédit rassemble les trente poèmes qui ont été publiés dans diverses revues. En plus de ces poèmes épars, qui apparaissent dans le recueil en version bilingue, l'ouvrage contient également quatre lettres, trois poèmes épistolaires (en version bilingue) et vingt-et-un articles écrits par Rachel au fil des ans. Près de la moitié de ces brefs articles ont vu le jour en 1929 et 1930, à la fin de l'existence de Rachel. Ils sont consacrés à la vie des pionniers, à la philosophie et à l'art - l'art plastique, la poésie, la littérature et le théâtre. Le traducteur, Bernard Grasset, a glissé ses introductions qui présentent chaque partie du volume. De plus, les parties du livre sont suivies par des notes de bas de page du traducteur ainsi que des notes regroupées pour chaque poème à la fin de la section des trente poèmes. Ces notes comprennent des informations supplémentaires sur l'arrière-plan et les éléments exprimés dans le poème ainsi que des explications sur la démarche de la traduction.

Les introductions du traducteur contribuent à la compréhension des états d'âme de la poétesse et de la situation existentielle dans laquelle elle se trouvait lors de la rédaction des textes. Le traducteur illustre les thèmes principaux qui ont occupé l'écrivaine dans les poèmes et les articles. Ceux-ci incluent l'attachement et l'enracinement à la terre, le travail agricole, la nature, le lac de Tibériade, le sentiment cosmique, la fraternité, le sens du sacré, et la soif de vivre. Dans l'introduction aux poèmes, le traducteur souligne les principales caractéristiques de la poésie de Rachel, de sa préoccupation avec la nature sauvage et le jardin cultivé, la relation entre le temps et l'espace, les émotions turbulentes et contrastées exprimées dans ses poèmes, et la musicalité de ses poèmes. Puis, la solitude, la mémoire et l'oubli, en répétant des symboles majeurs tels que la route, la lumière, le pont et le feu. Et enfin, son espoir essentiel même pendant sa maladie, et son lien avec les sources bibliques, comme dans le poème « La brebis du pauvre» (p. 61).

Pour sa connexion aux sources bibliques, le nom de ce recueil «Sur les rives de Tibériade » a été choisi : c'est le titre du premier de ses articles, écrit en russe à Odessa en 1919. Grasset le décrit comme "l'un de ses plus beaux articles, aussi empreint d'une coloration biblique » (p. 21), qui résume son existence et son œuvre. On peut citer dans ce contexte les propres mots de Rachel : «Ce n'est pas seulement un paysage, le lac de Tibériade, ni un fragment de nature - le destin d'un peuple s'allie à son nom. Avec des yeux sans nombre il nous regarde des profondeurs de notre passé, avec mille lèvres il parle au cœur » (p. 124).

La traduction des textes par Bernard Grasset est fidèle à l'original et conserve le style qui distingue Rachel. Le traducteur atteste lui-même dans l'introduction du recueil de poèmes en se référant à l'acte de traduction des poèmes de Rachel qu'il n'arrête pas de s'étonner de la façon dont « cette poésie écrite dans une langue très différente de la langue française se prête merveilleusement au passage en français, y chante comme naturellement» (p. 22).

Ce faisant, il résume l'impressionnant projet qu'il a commencé, en traduisant tous les écrits de Rachel en français. Le choix de produire une version bilingue des poèmes permet au lecteur bilingue de comparer l'original et la traduction. Malheureusement, la qualité d'impression de la version hébraïque dans tout l'ouvrage est médiocre, les lettres et les voyelles sont floues et rendent difficile la lecture fluide des poèmes en hébreu.

Cependant, c'est un volume varié, qui combine différents types de textes de la poétesse Rachel, en présentant la richesse de son œuvre et le fil conducteur entre ses différents écrits.

Ilanit Ben Dor-Derimian 
Ben-David Yaara, Une autre présence. Poèmes traduits de l'hébreu par Emmanuel Moses, Paris, Éditions Caractères (collection Planètes), 2019, 97 p. $18 €$.

Yaara Ben-David (née à Bagdad en 1947) est une poétesse et artiste israélienne renommée. À ce jour, elle a publié neuf livres de poésie et deux essais d'œuvres littéraires. Ses poèmes ont été traduits dans plusieurs langues et publiés dans des revues en Israël et à l'étranger. Elle a remporté des prix prestigieux et des bourses, comme le prix du Premier ministre au nom de Levy Eshkol et le prix Tchernichovski, poète israélien. Son livre Izoune Adine (en français «Un équilibre fragile ») réédité en 2016 a été retenu pour le fameux prix Brenner. La plupart des poèmes de ce recueil sont rassemblés et traduits en français dans «Une autre présence ». De plus, des dizaines de ses poèmes ont été mis en musique par des compositeurs israéliens.

Dans sa poésie lyrique autobiographique intime, souvent à la première personne, il y a une interaction entre le « je » et la réalité environnante, dans des cercles d'existence, d'espace et de temps qui se confondent. Ses poèmes présentent des rencontres entre le temps et le lieu, le personnel et l'universel, le passé et le présent, et plus encore. Elle joue entre les différents aspects de l'existence, lorsqu'un temps cache un autre temps, ou alternativement : un espace évoque les souvenirs d'un autre espace.

Le style d'écriture de Yaara Ben-David est délicat, riche en expression et profond dans la réflexion. Il est évident qu'elle est sensible à la mélodie des mots et qu'elle est inspirée non seulement par la tradition juive, mais par une vaste richesse culturelle et littéraire du monde entier. Elle mentionne dans ses poèmes des auteurs tels que Bruno Schultz («Éclats de temps », p. 74) et David Vogel («Limite », p. 28), l'artiste René Magritte (« Bruxelles $2016 »$, p. 73), et bien d'autres.

Par sa poésie, Yaara Ben-David est connectée à la réalité et l'observe à distance en même temps, à la fois personnellement et collectivement. D'une manière poétique, elle reflète ses sentiments à partir d'un traumatisme et d'une douleur suite à des épisodes personnels (notamment la mort de son frère), ou à des événements mondiaux, tels que les attentats terroristes à Paris en 2015 («Paris 2015 », p. 83) et à Bruxelles en 2016 (« Bruxelles 2016), p. 73), ou encore à la situation des migrants et des réfugiés en Europe («Le réfugié $\gg$, p. 84).

Yaara Ben-David fait preuve d'une grande virtuosité dans la création d'un collage verbal, similaire à l'art du collage visuel dans lequel elle excelle (ses collages sont exposés dans d'importantes galeries d'art et ont souvent un rapport avec sa poésie). Elle parvient à enrichir par la description littérale la réalité triviale des événements de la vie quotidienne. Cela peut être comparé à la façon dont un segment d'une image est agrandi à l'aide d'une loupe et tous les détails invisibles à l'œil nu sont révélés, ou comme la poétesse le décrit dans l'un de ces poèmes (« Image et voix en temps réel », p. 31):

Dans le tiroir se tapit le flash d'une photo cellulaire.

Agrandie, on y voit les pixels, des taches

Des points minuscules devenus profonds et plus grands,

Que je n'avais pas vus et que personne n'avait aperçus

[des poils secrets

[sur la petite image d'origine.

C'est la capacité poétique dont a été dotée la poétesse : d'agrandir l'image et descendre jusqu'à la profondeur des carrés des pixels.

Le deuxième fil conducteur de son travail est le désir humain de goûter à la fois de l'arbre de vie et de l'arbre de la connaissance, comme elle l'exprime dans certains de ses poèmes et notamment dans le poème «Un autre temps » (p. 15) :

Le temps se crée par le bond rapide des oiseaux originels

De l'arbre de la connaissance dans l'arbre de vie. 
Yaara Ben-David réalise ce besoin humain à travers la poésie qui relie l'arbre de la vie à l'arbre de la connaissance à travers les mots, faisant à la fois partie du monde abstrait et du monde réel.

Les poèmes dans ce recueil ont été traduits de l'hébreu vers le français par le traducteur Emanuel Moses (né à Casablanca en 1959), auteur d'une trentaine de livres de poésie, de nouvelles et de romans. En tant que traducteur, il a fait connaître des œuvres d'écrivains israéliens tels que David Grossman, S.Y. Agnon, Batya Gur, Yaacov Shabtaï et des poètes israéliens tels que Yehuda Amichaï. Il a également traduit des ouvrages de l'anglais et de l'allemand.

Le travail de traduction qu'il a effectué sur les poèmes de Yaara Ben-David s'illustre dans sa grande fidélité à l'original en hébreu et dans sa capacité à conserver le ton lyrique de l'œuvre originale et sa mélodie en hébreu et à les transmettre en français.

Ilanit Ben Dor-Derimian

Lessing Theodor, Jewish-Self-Hate, Berghahn, New York-Oxford, 2021, traduit et annoté par Peter C. Appelbaum, introduit par Sander L. Gilman, postfacé par Paul Reitter, édité par Benton Arnovitz, 186 p.

Né en 1872 à Hanovre au sein d'une famille juive acculturée, Theodor Lessing était un publiciste, essayiste et professeur de philosophie, démocrate convaincu, qui s'est battu dès le tournant du siècle pour les droits des femmes et l'éducation des couches défavorisées. Républicain engagé défendant avec un courage tant moral que physique une République de Weimar malade de ses faiblesses et de ses hésitations, Juif qui a combattu l'antisémitisme en Allemagne et plaidé pour un sionisme socialiste pour venir en aide aux Ostjuden, assassiné par des hommes de main des nazis le 30 août 1933 à Marienbad où il se pensait en sécurité, il est notamment passé à la postérité par cette expression de « haine de soi juive $»^{5}$. Son ouvrage, Der jüdische Selbsthass, paraît, aux éditions du Jüdischer Verlag, à la fin de l'année 1930, alors que l'Allemagne voit croître le nombre de députés nazis au Reichstag et, avec lui, l'antisémitisme se diffuser toujours plus profondément dans la société. C'est le dernier livre à être paru du vivant de Theodor Lessing, il est le fruit de la réflexion de toute une vie. L'avant-propos souligne : « L'auteur de ces lignes a lui aussi, dans sa jeunesse, connu une période d'adhésion exclusive à la 'germanité', de rejet absolu de la 'judéité' ». Der jüdische Selbsthass présente une série de portraits de six auteurs: Paul Rée (1849-1901), ami de Nietzsche et de Lou Andreas-Salomé ; le publiciste Maximilian Harden (1861-1927); Otto Weininger (1880-1903), auteur de Sexes et caractères (Geschlecht und Charakter) en $1903^{6}$; l'écrivain Arthur Trebitsch (1880-1927); le poète Walter Calé (1881-1904); Max Steiner (1884-1910), jeune scientifique qui se suicida à l'âge de vingt-six ans. Le propos central de Theodor Lessing à travers ces portraits, et les nombreuses digressions qu'il fait, est d'encourager les Juifs allemands à assumer pleinement leur judéité.

En France, Maurice-Ruben Hayoun traduisait et introduisait l'ouvrage de Theodor Lessing en 1990 chez Berg International sous le titre : La haine de soi-le refus d'être juif, traduction rééditée en 2011 avec une nouvelle postface - avec ce titre, MauriceRuben Hayoun évitait l'obstacle du concept «haine de soi juive». La traduction

\footnotetext{
${ }^{5}$ Voir à ce propos Martine Benoit, « Le phénomène de 'haine de soi juive' : de la douleur d'être Juif en Allemagne (1867-1933)», p.149-159, Cahiers d'études germaniques 2019/02 (nº77: Histoire des Juives et des Juifs d'Allemagne (1867-1933), études réunies par Laurence Guillon, Patrick Farges et Laurent Dedryvère.

${ }^{6}$ Sur Otto Weininger voir l'excellent livre de Jacques Le Rider, Le cas Otto Weininger : racines de l'antiféminisme et de l'antisémitisme, Paris, PUF, 1982.
} 
américaine, la première traduction en anglais de Der jüdische Selbsthass, a fait un autre choix : alors que jusqu'à présent, la littérature secondaire anglophone (notamment l'essai de Kurt Lewin en 1941 sous le titre "Self-hatred among Jews' "; 1'article de Lawrence Baron en 1981 « Theodor Lessing : Between Jewish Self-Hatred and Zionism »; le livre de Sander L. Gilman Jewish Self-Hatred: Anti-Semitism and the Hidden Language of the Jews en 1986) utilisait pour traduire le concept de « haine de soi juive » le terme selfhatred, pour la première fois, Peter C. Appelbaum et son éditeur Benton Arnovitz utilisent le mot « self-hate », en effet le plus proche du concept allemand de « Selbsthass » - et ce choix est à saluer.

Publiée avec l'accord et les encouragements des Éditions Suhrkamp, héritières depuis 1958 des éditions du Jüdischer Verlag fermées par les nazis en 1938, cette première traduction en anglais et cette nouvelle édition est à saluer fortement. L'ouvrage de Theodor Lessing, par une traduction très appliquée et un appareil de notes très complet, devient ainsi accessible au public anglophone. Tout a été fait pour laisser à la fois la texture allemande du texte et permettre au lecteur anglophone d'aujourd'hui de lire ce texte ardu.

Le traducteur Peter C. Appelbaum, professeur émérite de pathologie à l'université d'État de Pennsylvanie, finalise actuellement un travail sur la place des Juifs dans l'armée austro-hongroise (à paraître: Habsburg Sons : Jews in the Austro-Hungarian Army, 1788-1918, Cherry Orchard Books). Pour sa traduction, Peter C. Appelbaum s'est entouré de deux des plus grands connaisseurs du sujet: Sander L. Gilman, désormais professeur émérite, qui publie dès 1986 son Jewish Self-Hatred: Anti-Semitism and the Hidden Language of the Jews; Paul Reitter, directeur des écoles doctorales de l'université d'État de l'Ohio, germaniste spécialiste de la culture judéo-allemande, qui a travaillé sur Karl Kraus (The Anti-Journalist : Karl Kraus and Jewish Self-Fashioning in Fin-de-Siecle Europe, University of Chicago Press, 2008) avant de consacrer en 2012 une étude à la haine de soi juive, On the Origins of Jewish Self-Hatred (aux Princeton University Press) et d'écrire l'entrée « Selbsthass » (page 553-570) dans l'Encyclopédie de l'histoire et de la culture juives, dirigée par le grand Dan Diner et parue aux Éditions Metzler en 2015 (titre original : Enzyklopädie jüdischer Geschichte und Kultur).

Dans sa préface de quelque 25 pages, Sander L. Gilman évoque les destins et les engagements de Leon Pinsker, Jakob Wassermann, leur positionnement vis-à-vis de l'assimilation dans un contexte où l'antisémitisme en démontrait toutes les failles et les faiblesses. Sander L. Gilman revient sur le travail d'Anna Freud autour de la notion d'identification à l'agresseur, développe, à la suite de Kurt Lewin, la notion de haine de soi chez les Noirs américains. À la fin de son avant-propos, Sander L. Gilman insiste sur le fait que « ce que nous pouvons apprendre de la trajectoire du discours de la haine de soi, c'est qu'il pourrait bien s'agir d'un phénomène universel et complexe et qu'il ne s'agit certainement pas simplement d'un phénomène propre aux Juifs acculturés » (page 35). Dans sa postface, Paul Reitter rappelle quant à lui l'accueil du livre par les sionistes : directeur des Éditions du Jüdischer Verlag, Siegmund Kaznelson y voyait un coup de propagande sioniste bienvenu. Pour Paul Reitter cependant, il y a un abîme entre la réception du livre à sa sortie et sa réception aujourd'hui, un abîme qui pose question. Paul Reitter s'interroge en effet sur le moment de l'apparition du concept même de « haine de soi juive » sous la plume de Theodor Lessing et estime que celui-ci n'a pu apparaître qu'après la Première Guerre mondiale. Paul Reitter développe son propos à partir de textes du journaliste autrichien d'origine juive, Anton Kuh, qui utilisa l'expression de « haine de soi juive » dans des discours des années 1918-1920, discours publiés à Berlin en 1921 aux éditions Erich Reiss sous le titre Juden und Deutsche (Juifs et Allemands) ${ }^{7}$.

\footnotetext{
${ }^{7}$ Une réédition de ce texte est parue sous la direction d'Andreas Kilcher en 2003 aux
} Éditions Böhlau de Vienne. Anton Kuh a été redécouvert au début des années 2000, ses 
Selon Reitter, Anton Kuh et Theodor Lessing devaient se connaître, ils travaillaient tous les deux pour le quotidien Prager Tagblatt, journal des démocrates de langue allemande en Tchécoslovaquie. Anton Kuh aurait introduit le terme de haine de soi notamment pour son approche psychologisante et son rapport au conflit père-fils. Le propos de Paul Reitter est de confronter les textes d'Anton Kuh au propos de Theodor Lessing.

Le texte de Theodor Lessing est, comme le souligne Peter C. Appelbaum dans son introduction, un livre sur la nature complexe de la haine de soi des Juifs allemands de la fin du XIX ${ }^{\text {ème }}$ siècle jusqu'à la République de Weimar. Peter C. Appelbaum souligne les difficultés rencontrées devant un texte sinueux, comportant de nombreuses erreurs que Peter C. Appelbaum corrige avec beaucoup de doigté par son impressionnant appareil de notes : alors que le livre de Theosor Lessing comportait 17 notes de son auteur, la traduction en comporte plus de 300. Le choix éditorial a été de placer ces notes à la fin de chaque chapitre: l'appareil de notes distingue les notes de Theodor Lessing, reproduites en italique, des notes de Peter C. Appelbaum. Placer les notes en fin de chaque chapitre permet aux lecteurs d'accéder rapidement à ces notes ; cela coupe un peu peutêtre la lecture du texte de Theodor Lessing. Ces notes sont cependant indispensables pour une lecture intelligente et abordable à un lecteur d'aujourd'hui : Peter C. Appelbaum y rappelle ce qu'étaient la Jewish Agency, le Bilu, la Légion juive, donne des explications sur la vie juive, explique des mots de yiddish ou d'hébreu, donne une courte présentation des grands noms que cite Lessing (de Agnon à Moses Hess, Leo Pinsker, Martin Buber, en passant par Moses Mendelssohn, Heinrich Heine, Gotthold Ephraim Lessing, Johann Wolfgang Goethe, Friedrich Schiller, Hugo von Hofmannsthal, Franz Werfel, Jakob Wassermann, Karl Kraus mais aussi Ferdinand Lassalle, Karl Marx, Friedrich Nietzsche, Arthur Schopenhauer, Sigmund Freud, Lou Andreas-Salomé, mais aussi les auteurs antisémites tels Paul de Lagarde, Julius Langbehn, Eugen Karl Dühring, Houston Stewart Chamberlain). Peter C. Appelbaum fait montre d'une profonde intégrité scientifique en n'hésitant pas à admettre dans une note qu'il n'est pas suffisamment qualifié pour expliquer tel ou tel passage (voir ainsi note 23 p. 154, sur un passage de Theodor Lessing où celui-ci confronte la pensée de Paul Rée à celle de Friedrich Nietzsche). Pour certains mots, le traducteur a préféré garder le terme allemand, choix qu'il explique en notes : ainsi des termes « Volk » ou « völkisch », « intraduisibles en anglais » (note 26 p. 155 ou 18 p. 237).

Cette nouvelle traduction permet de mieux comprendre aujourd'hui le texte de Lessing, souvent complexe et obscur. On ne peut donc que saluer ce double travail de traduction et d'éditorialisation - et encourager les bibliothèques universitaires françaises à faire l'acquisition de cette première traduction anglophone.

Martine Benoit

Berest Anne, La carte postale, Paris, Grasset, 2021, 512 p. $24 €$.

Anne Berest, romancière, auteure (elle a consacré entre autres un livre à Françoise Sagan en 2014 et un autre - coécrit avec sa sœur Claire - à son arrière-grand-mère, Gabriële, épouse de Francis Picabia en 2017) a beaucoup fait parler d'elle cette année...

D'abord parce que son livre a figuré sur la liste finale pour le Goncourt, qu'il a été encensé par quelques critiques. Parce qu'ensuite il a été éreinté sans ménagement par Camille Laurens dans les colonnes du Monde.

Le récit commence le 6 janvier 2003 par la réception dans la boite aux lettres de la mère de l'auteure d'une carte postale, datant des années quatre-vingt-dix et comportant les

œuvres complètes publiées par les Éditions Wallstein en 2016 en sept volumes sous la direction de Walter Schübler. 
seuls prénoms des grands-parents, oncle et tante de la mère d'Anne : Ephraïm, Emma, Noémie et Jacques. Tous sont morts assassinés à Auschwitz en 1942. Ni signature ni explication... Mauvaise plaisanterie, menace ?

L'épisode est oublié quand dix ans plus tard, séjournant chez sa mère avant d'accoucher, Anne Bérest l'interroge.

C'est le début d'un long récit, objet du livre $1^{\text {er }}$, intitulé « Terres promises ».

Cette histoire commence en Russie avec Ephraïm Rabinovitch, arrière-grand-père de l'auteure. Il quitte la Russie en 1919 avec sa femme, s'installe pour un temps à Riga (en Lettonie) où naît Noémie, en 1923 ; ils émigrent en Palestine puis s'installent enfin à Paris. Assimilation... Les enfants fréquentent les bons lycées parisiens, Ephraïm devient Eugène Rivoche, demande la nationalité française - qui lui est refusée - achète une maison de campagne en Normandie.

Mais les menaces se précisent: Hitler est devenu chancelier, la Tchécoslovaquie est annexée. La guerre est déclarée avec les lois anti-juives, l'exposition «Le Juif et la France $\gg . .$.

Myriam épouse Vincente (fils de Francis Picabia), échappe miraculeusement aux Allemands. Mais Jacques et Noémie sont arrêtés, emprisonnés à Evreux puis à Pithiviers avant d'être déportés à Auschwitz. Jacques est gazé. Noémie meurt peu après du typhus. Emma et Ephraïm sont arrêtés à leur tour, transférés à Drancy et gazés à Auschwitz le 7 novembre 1942.

La deuxième partie, intitulée « Souvenirs d'un enfant juif sans synagogue », est consacrée aux interrogations de l'auteure, à ses conversations avec sa mère, à la poursuite de l'enquête (elle a fait appel à une agence de détectives privés, à un graphologue), à ses recherches en Normandie (dans la maison de campagne de ses parents).

Les troisième et quatrième parties sont consacrées à Myriam, la grand-mère de l'auteure, qui seule a échappé à la tragédie familiale. C'est l'occasion d'un (trop) long récit sur leur vie en Provence, au trio amoureux qu'elle constitue avec son mari et Yves, un ami. On y croise René Char, on continue de travailler pour la Résistance. Puis après le Débarquement, la libération de Paris, la naissance de Lélia (mère de l'auteure), les déportés reviennent. Myriam attend à l'hôtel Lutetia l'improbable retour de ses parents. En vain. Elle part pour l'Allemagne, à leur recherche. Elle confie sa petite fille à une vieille nourrice, en Provence. Après quelques années, elle s'y installe à son tour, perd peu à peu la mémoire.

À l'occasion de son quarantième anniversaire, plutôt qu'un séjour vinicole et une exposition en Provence, les souvenirs la rattrapent. Elle se rend à Céreste où la petite Lélia avait été mise en nourrice. C'est Mireille, une vieille femme de 90 ans, qui lui donnera la clef de l'énigme de la carte postale...

Si l'on passe sur la polémique déclenchée par l'article de Camille Laurens (qu'importe la cuisine littéraire, l'article est argumenté, rosse et souvent juste à mes yeux), l'ouvrage devrait laisser perplexes les lecteurs : il ne s'agit ni d'Histoire ni de témoignage même si Anne Berest s'est appuyée sur une vaste documentation.

Mais elle y mêle inventions personnelles, lettres privées, monologues et dialogues - avec sa fille, sa mère, son compagnon - réflexions diverses (sur l'antisémitisme) et élucubrations (elle croit aux actes psychomagiques et aux "poches de temps", cite à l'appui de ses dires Jodorowsky).

Un critique a inventé pour qualifier ce livre les mots de «thriller mémoriel », création curieuse et assez indécente au regard des sujets abordés. Le livre est aussi semé de pastiches, volontaires ou non (Giono et la vie provençale); le trio amoureux, souvenir de Jules et Jim de Pierre-Henri Roché ? L'auteur y abuse du name dropping (Irène Némirovsky, Walter Benjamin, Beckett, etc). 
Tout ceci me paraît souvent irritant et déplacé en regard du sujet (la Shoah) ou de ce que l'auteure prétend avoir voulu mener (je cite la quatrième de couverture, c'est-à-dire une « quête initiatique sur la signification du mot « Juif» dans une vie laïque »).

L'amateur de romans retournera à Gary ou Modiano par exemple. L'amateur de littérature se procurera l'ouvrage opportunément consacré par la Pléiade aux écrits des camps (Robert Antelme, Charlotte Delbo, Jorge Semprun, Jean Cayrol, Piotr Rawicz), paru en octobre dernier.

Fabrice Danon

Rota Olivier, Les catholiques anglais et la "question juive» (1917-1967). D'une approche politique à une approche spirituelle, Paris, éd. du Cerf, juin 2021, 656 p. $39 €$.

Auteur ou directeur de plusieurs ouvrages sur les relations inter-religieuses, Olivier Rota a soutenu en novembre 2019, à l'université de Reims, une habilitation à diriger des recherches. L'ouvrage qui vient de paraître, deux ans après, est la publication de son épais mémoire de soutenance. Il est actuellement maître de conférences à l'université catholique de Lille.

Une recherche initiale, et bien antérieure, sur l'antisémitisme des catholiques en GrandeBretagne, qui se double souvent d'une réflexion philosophique bien que l'auteur se défend d'être un historien des idées, s'est développée en une recherche sur les relations inter-religieuses pour aboutir à une étude substantielle sur les catholiques anglais et la « question juive ». La focalisation sur les catholiques mérite d'être retenue dans la mesure où ceux-ci constituent, en Angleterre, une minorité tout comme les Juifs qui les indisposent. Et ceci à la différence de la France, ou d'autres pays européens, où les catholiques sont majoritaires et dont la civilisation intègre les valeurs catholiques. Pour ses nombreux travaux constitutifs de son mémoire d'habilitation à diriger des recherches, Olivier Rota n'a pas ménagé sa peine, se rendant en Israël pour consulter des archives (Notre-Dame de Sion, Assomptionnistes, patriarcat latin etc.) ou à Rome, et principalement en Angleterre où il fréquenta les sœurs de Sion et d'autres milieux catholiques impliqués dans le dialogue entre Juifs et catholiques. Ce qui explique l'abondance des sources archivistiques, littéraires et de la presse. Près d'une trentaine de centres d'archives ont été visités et pas moins d'une cinquantaine de journaux ou revues ont été utilisés !

Une bibliographie non moins abondante laisse une large part aux publications en anglais et l'auteur ajoute une bibliographie pour chacun des huit chapitres qu'il rassemble en un appareil critique. La présence d'un index des noms de personnes est bien appréciable. Non négligeables également sont les annexes livrant des documents pour la plupart en anglais.

En outre, il faut souligner une écriture agréable à lire, le style est alerte et le vocabulaire est riche. Les arguments sont toujours exposés avec des références archivistiques ou bibliographiques grâce à de nombreuses notes qui informent aussi sur l'historiographie.

Dans son introduction, Olivier Rota invite à définir les termes tant il est vrai que le sens de certains d'entre eux varie selon le contexte politique et social. À commencer par « catholiques anglais » et la " question juive», la judenfrage des Allemands qui évolue en «question de l'État d'Israël», qui sont autant d'expressions méritant d'être bien précisées. Et plus encore les termes de «mission», «apostolat» sont à comprendre différemment selon les années. Mais tout cela Olivier Rota l'explique fort bien : le but de la mission passe de la conversion au dialogue, à l'échange. Cette évolution n'est d'ailleurs pas le fait des seuls catholiques de Grande-Bretagne.

Le choix des bornes chronologiques est judicieux. L'année 1917 est, en effet, riche en événements décisifs pour l'Europe en guerre mais aussi pour, et surtout pour, la Grande- 
Bretagne. En 1917, rappelle Olivier Rota, la victoire des Bolcheviks bouleverse le champ religieux et va repositionner les catholiques, de Grande-Bretagne et d'ailleurs, vis à vis des Juifs que l'on assimile bien vite à l'athéisme communiste antichrétien. 1917 c'est encore, pour la Grande-Bretagne, la pénétration militaire en Palestine, avec l'entrée des troupes d'Allenby dans Jérusalem, et la lettre que Balfour envoie à Lionel de Rothschild l'assurant du soutien du Gouvernement de Sa Majesté pour l'établissement d'un foyer national juif en Palestine. Les bases d'un développement sioniste sont alors jetées, ce qui va inviter les catholiques à revoir leur relation avec les Juifs qui désormais sont supposés prendre possession des lieux saints. Bref, le choix de l'année1917 est tout à fait pertinent pour borner cette étude. Et celui de 1967 l'est tout autant : le concile du Vatican II vient de se terminer avec la déclaration Nostra AEtate qui proscrit l'antijudaïsme. C'est aussi l'année de la guerre des Six-Jours qui change la vision que l'on peut avoir d'Israël et, en conséquence, des Juifs.

On peut relever quelques idées fortes.

- En premier lieu vient le parallèle tout à fait recevable entre catholiques irlandais et Juifs, en tant que minorités religieuses discriminées en Angleterre et plus précisément dans l'East End londonien où ces communautés connaissent un peuplement dense. Et la présence d'un Zangwill n'a pas été oubliée tant pour sa lutte contre l'antisémitisme que pour son soutien à la cause sioniste. Cette particularité anglaise du catholicisme méritait en soi une étude approfondie. Encore que, dans un autre contexte de libération nationale, les Italiens s'habillaient, eux aussi, des habits des Hébreux gagnant la Terre Promise (Verdi) tout comme les Irlandais dans leur imaginaire se voyaient dans la peau de Juifs.

- Les pages sur l'antisémitisme des penseurs catholiques anglais, tels Belloc et les frères Chesterton, sont particulièrement fortes. On pourrait y voir l'influence des catholiques français, notamment pour Belloc. Car, comme le précise Olivier Rota, l'antisémite catholique anglais développe les mêmes arguments que le catholique français pétri d'antisémitisme.

- La « question juive » finit par devenir « la question du sionisme » et ceci dès les années 1930 puisque la Grande-Bretagne a hérité d'un mandat sur la Palestine. Dans la pensée catholique, en Angleterre et ailleurs, le sionisme se teinte d'antichristianisme. L'évolution sémantique des termes finit par engendrer une évolution politique et religieuse du positionnement des catholiques. Or parallèlement, la persécution des nazis préoccupe les catholiques, ainsi apparaissent et se développent des mouvements favorables aux Juifs comme le CCJ (Council of Christians and Jews) pour ne citer que lui. Cette contradiction a bien été analysée par Olivier Rota.

- Autre idée forte d'importance : le rôle efficace des Juifs convertis au catholicisme dans la nouvelle approche de la mission envers les Juifs, qui se transforme en dialogue. Pour ne citer que les plus connus : Mgr Osterreicher, mais encore Mgr Baum, et aussi quelques sœurs de Sion, ou encore des laïcs comme Mme Marinoff. Tout comme la présence des dominicains dans ce changement. On est loin des Liberman et des Ratisbonne du XIX siècle qui n'avaient que la conversion à offrir à leurs anciens coreligionnaires. Rappelons au passage qu'en France et en Palestine-Israël, on retrouve aussi des Juifs convertis au catholicisme et des dominicains aux premiers rangs des partenaires catholiques du dialogue inter-religieux.

- La définition de l'œcuménisme occupe aussi plusieurs paragraphes, à juste titre tant il est vrai que sa limitation stricte aux relations intra-chrétiennes fut, et est encore, discutée, parfois âprement.

- Les progrès du dialogue judéo-catholique ont leur place dans l'étude de l'après-guerre. Les pages sur l'influence de Jules Isaac, le fondateur de l'Amitié judéo-chrétienne en France en 1948, me paraissent complètement neuves, de même celles sur la présence anglaise à la conférence de Seelisberg puis de Fribourg. Elle-même avait été précédée par la conférence d'Oxford qui prépare les réflexions à mener. En France, les catholiques 
manifestent, au tout début, une certaine méfiance face à ce mouvement inter-religieux, il semble qu'en Angleterre, avec la conférence d'Oxford, les craintes ne soient pas de mise. - En ce qui concerne les pages sur le concile du Vatican II, on peut regretter la mise à l'ombre de la présence des sœurs de Notre-Dame de Sion dans les coulisses. Elles ont beaucoup agi en sous-main mais peut-être n'y avait-il pas beaucoup de religieuses anglaises parmi elles. En revanche il faut reconnaitre le bien-fondé du rappel du contexte des années 1960 avec l'affaire de la pièce de théâtre Le Vicaire, qui d'ailleurs secoua aussi la France, et avec le procès Eichman qui bouleversa la population israélienne et juive dans le monde mais aussi les non-Juifs et au premier chef les catholiques. Ces événements ont pu avoir un écho dans les couloirs du Vatican.

Après la lecture, il reste à se réjouir d'une telle publication très bien documentée et qui renouvelle très certainement l'histoire de certaines étapes du dialogue entre catholiques et juifs qui évolue de l'animosité à l'amitié.

Danielle Delmaire

Fajnkuchen Franck, Yzkor. Une famille juive en France entre 1940 et 1944, Beaumontois-en-Périgord, Les éditions Secrets de Pays, 2021, 182 p. $20 €$ (préface d'Élisabeth Badinter, postface de Bernard Revirego).

Yzkor est un terme hébreu bâti sur une racine qui évoque le souvenir. Sous cette forme, le mot est un verbe conjugué au futur qui équivaut à un impératif, une injonction. Il s'agit surtout du titre d'une prière prononcée en souvenir des défunts. Et c'est bien à cela que Franck Fajnkuchen nous invite: nous souvenir d'une ou plutôt de familles juives disparues dans la Shoah ou qui ont frôlé la mort dans l'État français entre 1940 et 1944. À partir de sa propre famille, de ses grands-parents paternels et d'autres parents par un jeu d'alliances matrimoniales, F.F. développe les parcours de familles issues de trois couples mais cette «tribu familiale » s'étend sur onze familles! C'est dire l'étendue de la recherche de l'auteur qui s'appuie sur les récits familiaux, nombreux entre cousins et cousines, et sur des documents d'archives abondants et aussi sur deux ouvrages d'historiens confirmés, indispensables pour étudier le sort des Juifs de Lens, ville que ces familles ont quittée pour se réfugier dans le Périgord, celui de Nicolas Mariot et Claire Zalc et celui de Bernard Revirego (d'où la postface élogieuse de ce dernier, archiviste du département de la Dordogne $)^{8}$. Trois piliers indispensables pour toute recherche sérieuse en histoire contemporaine: les archives, la mémoire des témoins et les études d'historiens.

Bien que non historien de métier, F.F. a le souci de référencer correctement ses sources archivistiques et de restituer les récits qu'il a pu recueillir. Toutefois, la date de l'entretien fait défaut et le lecteur aimerait savoir si l'entretien est récent, donc éloigné des faits, ou plus ancien, donc livrant un récit moins brouillé par le temps. Il n'empêche que ces récits constituent une source bien riche pour reconstituer ce passé.

D'emblée, il faut souligner les grandes qualités pédagogiques de F.F. Tout d'abord, idée excellente : l'ouvrage est accompagné d'un carton mobile, que le lecteur peut avoir sous les yeux à tout moment, étalant la généalogie des trois couples fondateurs des onze familles et, plus appréciable encore, chaque lignée issue des trois couples apparaît d'une couleur différente attribuée à chaque couple d'origine. Il faut reconnaitre que ce carton est bien utile pour suivre les membres de la «tribu familiale» car les alliances

\footnotetext{
${ }^{8}$ Nicolas Mariot et Claire Zalc, Face à la persécution : 991 Juifs dans la guerre, Paris, éd. Odile Jacob, 2010 ; Bernard Revirego, Les Juifs de Dordogne, 1939-1944: de l'accueil à la persécution, Périgueux, éd. Fanlac, Archives départementales de la Dordogne, 2003.
} 
matrimoniales sont serrées (deux sœurs épousent deux frères issus d'un autre des trois couples) et étendues. Autre initiative appréciable : les premières pages présentent les trois couples souches (les Fajnkuchen, les Timberg, et les Biezunski-Dembinski, avec les photos-portraits) avec leurs enfants qui constituent les familles suivies dans cette saga. De même les dernières pages développent des notes biographiques concernant, cette fois, toutes les personnes dont F.F. suit le parcours pendant la guerre, soit environ 70 personnes. Et enfin, un «tableau de synthèse» récapitule les dates de naissance et d'arrivée en France, le lieu de résidence ou de passage de la cinquantaine de personnes. Grâce à ces outils, il est impossible de s'égarer dans cette généalogie foisonnante.

Autre outil pédagogique très utile pour connaître la dispersion des familles dans le Périgord : la carte qui, en p. 61, localise les lieux de résidence plus ou moins cachée des familles. Celles-ci sont dispersées mais elles ne sont pas très éloignées (toutefois l'absence d'échelle ne permet pas de mesurer les distances) afin de rester en contact si nécessaire.

Fort de la connaissance des récits familiaux, l'auteur débute son ouvrage par une analyse de ses découvertes. Il restitue son itinéraire pour retrouver les traces des persécutés qui se cachent. Il déplore néanmoins ses échecs non pas dûs à une inadvertance de sa part mais parce que les archives et les témoignages ne mentionnent plus certains membres des familles : ce sont les « évaporés ». C'était bien d'ailleurs l'intention des bourreaux qui marquaient les détenus du qualificatif Nacht und Nebel, qui devaient disparaître physiquement et mémoriellement. Parmi ces « disparus » et ces " évaporés », figure Manek Fajnkuchen, le grand-père de Franck que celui-ci suit jusqu'à son arrestation en 1944 mais dont les traces se dispersent même en ce qui concerne son convoi de déporté et son sort à Auschwitz. Et le témoignage d'un rescapé (Nathan Alpern) ne parvient pas à faire la lumière.

Dans cette analyse, préalable au récit des voyages et déménagements, F.F. constate l'implication de l'administration française dans cette chasse aux Juifs : les préfectures et les mairies correspondent pour livrer des informations qui finissent par aboutir sur le bureau d'un policier en charge des arrestations. Face à la menace qui pèse de plus en plus, les chefs de ces familles pressentent la gravité du danger : «Ces éléments ont forgé ma conviction que les parents connaissaient le risque mortel d'une arrestation, pour eux comme pour leurs enfants » (p. 28). Après les rafles de l'été 1942 qui touchent les Juifs de la zone non occupée, donc aussi le Périgord, la stratégie adoptée par ces familles est la dispersion du noyau familial et/ou un domicile changeant : « Jacques et Rose Biezunski se séparent de leurs enfants » (p. 26) et «Sarah Fajnkuchen et sa famille [...] décident chaque nuit de trouver refuge dans un logement à l'adresse tenue secrète » (p. 26). Quant à la survie, elle dépend de la solidarité entre les familles mais aussi des voisins.

Après cette analyse pointue, F.F. en vient au récit des pérégrinations de toute «la tribu familiale » à partir de l'émigration depuis la Pologne jusqu'à la Libération.

Le berceau familial est donc la région de Kielce et de Cracovie. L'établissement dans le Nord et le Pas-de-Calais se fait pour quelques-uns via la Belgique ou via l'Allemagne et la Lorraine, voire la Palestine qui ne fut qu'une étape. La plupart de ces couples pratiquent le commerce sur les marchés de la région lensoise qui a accueilli un grand nombre de Polonais non Juifs entre les deux guerres. L'origine commune facilite les échanges entre ces deux populations venues de Pologne. Les enfants intègrent l'école de la République et l'intégration se réalise facilement. Au point que lorsque la guerre est déclarée l'engagement des pères de ces familles juives dans les Régiments de Marche des Volontaires Étrangers est massif. Tous se mobilisent pour défendre une nouvelle patrie qui semble bien les avoir adoptés.

La brutale défaite de la France face aux forces allemandes bouleverse le sort de la « tribu familiale». Pour les Fajnkuchen c'est la fuite : d'Avion vers la Dordogne où les rejoignent d'autres familles parentes. De juin 1940 à août 1942, mois des rafles dans la 
zone non occupée, le séjour dans le sud se déroule plus ou moins paisiblement. Mais après les rafles, la dispersion s'impose, les cachettes doivent être plus sécurisées et moins visibles. Pour toutes ces familles, les années 1943-1944 sont les plus difficiles car le danger est quotidien. Pour certains c'est l'errance, jusqu'à Lyon avec une tentative de passer en Suisse. Pour d'autres, adultes et adolescents comme Georges Biezunski, c'est l'engagement dans la résistance.

Une affaire pénible bouleverse les familles Fajnkuchen et Biezunski : la condamnation à mort de leur amie Huguette Conquet qui, tout en travaillant pour la milice, aidait ses amis juifs !

Les citations de Georges Biezunski, en exergue de quelques chapitres résument l'évolution du sort de la « tribu familiale » : pour 1939-1940 « J'ai vu les Stukas attaquer en piqué »; pour le séjour en Dordogne de juin 1940 à août 1942 : « On a connu une période assez tranquille »; de l'été 1942 à février 1943 : « Nous étions dans le désespoir le plus total $[\ldots]$ ». Et au retour : «Il n'y a plus rien à la maison, tout est vidé » selon François Dembin.

Il faut encore ajouter que l'auteur a apporté des preuves en publiant de nombreux documents d'archives, très bien référencés et des photos.

Conclusion de cette saga familiale : les Juifs ont participé au combat des forces françaises dans la Résistance, ils ont aussi résisté en fuyant avec témérité et ténacité la persécution. Fuir, se cacher, vivre coûte que coûte dans la clandestinité est déjà une forme de résistance au programme d'extermination des Juifs mis en place par les nazis et appliqué par leurs supplétifs français. Enfin, les Juifs ont pris leur sort en mains : ce sont eux qui décident de résister à la persécution et ce sont eux qui contribuent à leur propre survie même s'il leur a fallu de l'aide de la part de non-Juifs.

Il faut ajouter que grâce aux recherches de Monique Heddebaut, aux archives départementales du Pas-de-Calais (AD 62), on en sait un peu plus sur Joseph Fajnkuchen, le frère de Manek donc le grand-oncle de Franck Fajnkuchen. Le 2 février 1940, alors qu'il va avoir 43 ans, il se présente au Conseil de révision à Arras, pour s'engager dans l'armée, c'est-à-dire les Régiments de Marche des Volontaires Étrangers (RMVE). Le dossier renseigne sur son parcours d'immigré venu de Pologne au lendemain de la Première Guerre mondiale : il est arrivé en France en 1920 et après de nombreuses étapes en Moselle, en Ardennes, à Lille, à Douai, à Lens et Lambersart (près de Lille) il s'établit à Liévin en 1934 et en 1940 il y réside toujours. Il est alors tailleur. Selon le commissaire de police qui rédige les fiches de signalement : « nous n'avons rien à lui reprocher » quant à sa moralité, ses mœurs et sa position politique. Ce dossier qui repose aux $\mathrm{AD}$ 62, à la cote M 6855 complète et confirme le témoignage de la fille de Joseph p. 45-46 du livre.

Danielle Delmaire

Bande Alexandre, Biscarat Pierre-Jérôme et Lalieu Olivier (dirigé par), Nouvelle histoire de la Shoah, Paris, Passés composés, août 2021, 413 p. $24 €$.

Vingt contributions composent ce volume dont le titre annonce une réécriture de l'histoire de la Shoah, en France et dans toute l'Europe et même de la persécution des Tsiganes puisque Ilsen About présente le « génocide et [les] persécutions des Roms et des Sinti en Europe 1933-1946». Elles ont été rassemblées par des historiens confirmés de la Shoah : Alexandre Bande est expert auprès de la Fondation pour la Mémoire de la Shoah et intervient au Mémorial de la Shoah, Pierre-Jérôme Biscarat fut responsable pédagogique pour la Maison d'Izieu, mémorial des enfants juifs exterminés, et a travaillé jusqu'en 2020 avec l'association Yahad-In Unum qui met en évidence la Shoah par balles dans l'Europe orientale, Olivier Lalieu, auteur de plusieurs ouvrages sur la mémoire de la Shoah, est responsable de l'aménagement des lieux de mémoire et des projets externes 
du Mémorial de la Shoah et membre du Centre international pour l'éducation sur Auschwitz et l'Holocauste au musée d'État d'Auschwitz-Birkenau. L'ouvrage est dédié à Serge et Beate Klarsfeld qui ont «inspiré, guidé et soutenu» les travaux de ces historiens.

La vingtaine d'études présentée dans cet épais volume de plus de 400 pages couvre toute l'Europe, soit tout l'espace de la mise en œuvre de la Shoah, et certaines d'entre elles se placent dans la perspective d'autres génocides. Elles se répartissent en quatre parties.

Première partie : Origines, étapes et géographie du processus génocidaire.

Elle expose le contexte particulièrement en Europe centrale et orientale. Johan Puttemans situe l'origine du processus génocidaire dans l'extermination, dès la fin de la décennie 1930, des handicapés physiques et mentaux. L'auteur renouvelle effectivement la compréhension des meurtres collectifs en soulignant le lien entre extermination des handicapés et disparition des Juifs et des Tsiganes, jugés eux aussi asociaux et « inutiles ». Les procédés sont communs : chambres à gaz, injections létales effectuées secrètement, les traces doivent aussi disparaitre.

Il revenait à Tal Bruttmann d'étudier la « mise en place de la 'Solution finale' ». Il insiste sur le « tournant de l'été 1941 ». Trop souvent l'origine de l'extermination des Juifs est datée avec la conférence de Wannsee (20 janvier 1942) mais les tueries de Juifs par les Einsaztgruppen, la mise à mort des prétendus « judéo-bolcheviques », les pogromes en Europe (Jedwabne) etc. sont antérieurs. « À la mi-septembre 1941, Hitler ordonne que le territoire de l'Allemagne soit débarrassé de toute présence juive» (p. 40). L'article est remarquable pour ses notes abondantes et largement historiographiques.

Les trois articles suivants présentent les procédés de l'extermination : la mise à mort dans les ghettos (Audrey Kichelewski), les tueries de masse avec la "Shoah par balles » (Andrej Umansky et Patrick Desbois), les «centres de mise à mort» (Christophe Tarricone). La carte couverte de points figurant les ghettos (p. 50) montre que ces structures de concentration humaine furent excessivement nombreuses et, finalement, d'une grande diversité. D'où l'importance de définir les termes car quels points communs unissent le ghetto de Varsovie et le quartier de Salonique réservé aux Juifs, appelé aussi ghetto et qui fut plutôt un camp de transit? Les conditions de vie ou de survie varient selon les lieux d'implantation et les époques. De même Christophe Tarricone s'attache à définir le terme de «centre de mise à mort » qui « rend compte du sort spécifique des Juifs » dans les pratiques répressives des nazis. Ce centre peut être une chambre à gaz ou bien le lieu d'une fusillade de masse comme à Ponar, près de Vilna. Il peut évoluer aussi avec les mois pour devenir un camp entouré de barbelés et, dans un souci de rationaliser la mise à mort, ce camp est toujours installé près des routes et des voies ferrées. Ici, comme souvent ailleurs, la discrétion impose la destruction des corps. Après quinze années de prospection en Europe orientale, Patrick Desbois et son équipe est parvenue à définir cinq étapes dans le déroulement d'une exécution par balles : le rassemblement des victimes, leur déplacement, leur déshabillage, la fusillade et enfin le dépouillement. Là encore, les assassins effacent les traces autant pour masquer les crimes que pour tromper le voisinage qui pourtant parfois participe à la curée. Il faut remercier Umansky et Desbois pour l'exposé de leur méthodologie, dans leurs notes.

Avec la Pologne, les territoires soviétiques furent particulièrement touchés par les massacres mais la prise de conscience du danger par les victimes elles-mêmes est à l'origine des sauvetages, sujet étudié par Marie Moutier-Bitan. L'auteure dresse la carte des lieux de sauvetage car si les Juifs prennent eux-mêmes la décision de se cacher, ils ont besoin d'aides pour survivre, même dans les forêts. Les sauvetages ne réussissent pas toujours et les échecs sont parfois occultés par l'image du Juste sauveteur.

Cette première partie se clôt par une heureuse contribution de Ilsen About sur le génocide des Roms et des Sinti qui commence dès 1933. Parfois « des Roms partagent le sort des 
Juifs dans des ghettos ou dans des camps » (p. 134), dans les territoires conquis par les Allemands. Les massacres et les asphyxies par le gaz sont le lot commun.

Seconde partie : la Shoah en France.

Quatre auteurs ont été invités à faire part des nouveaux travaux sur la Shoah en France. Toutefois, André Kaspi s'en tient à des recherches peu renouvelées sur l'engagement des Juifs dans la résistance à la politique d'extermination dans la mesure où ses références bibliographiques se cantonnent à la fin du siècle dernier. Il analyse la présence des Juifs dans les différents mouvements de résistance, dans les maquis (juifs parfois), dans les rangs gaullistes etc. Quant à Cindy Biesse qui brosse un tableau de l'aide et des sauvetages accordés aux Juifs, elle a raison de diviser la chronologie en un avant et un après l'été 1942 durant lequel les rafles sont les plus nombreuses. Elle rappelle, à juste titre, l'engagement des mouvements non juifs comme la CIMADE et juifs comme l'OSE, les Éclaireurs israélites de France, le Joint. Elle élabore une sociologie des sauveteurs, comme a pu le faire Jacques Sémelin', et pointe l'importance des cheminots pour évoquer leur implication dans le sauvetage des Juifs en gare de Lille-Fives mais ses informations sont maladroites et jamais ces cheminots ne sont parvenus «à extraire une centaine d'enfants des wagons à destination des centres de mise à mort » (p. 207). En revanche ils ont accompli l'exploit de sauver une soixantaine de personnes, adultes et enfants ${ }^{10}$.

Laurent Joly fait le point sur la politique du Gouvernement de Vichy envers les Juifs et liste toutes les décisions : statuts, recensements, « aryanisation » etc. Il établit le parallèle entre la politique allemande et la politique de Vichy pour conclure qu'elles se complètent parfaitement. À propos de la rafle du Vel d'Hiv, il rappelle qu'elle fut globalement un échec dans la mesure où, dans la grande majorité des arrondissements de Paris, le taux d'arrestation est inférieur à $50 \%$ du nombre des personnes à arrêter. Et il invite, comme le font la plupart des historiens maintenant, à en finir avec «le cliché des victimes passives », car si la rafle échoue c'est que les Juifs lui résiste en se cachant.

Thomas Fontaine apporte une chronologie, bien établie désormais grâce aux travaux récents dont il fait état dans ses notes abondantes, de la déportation des Juifs de France. La première étape touche les Juifs communistes, déportés par représailles ${ }^{11}$, puis vient le tournant de juin et juillet 1942 avec les grandes rafles responsables du surpeuplement des camps de rassemblement. C'est durant l'été 1942, se prolongeant jusqu'à novembre, qu'est réalisée plus de la moitié du programme de la « Solution finale » en France. Par la suite, la machine s'enraye et les convois sont de plus en plus espacés. L'auteur insiste sur les moyens de la déportation : les voies ferrées, les gares et la réquisition du personnel de la SNCF.

Troisième partie : Enjeux mémoriels et éducatifs.

C'est, me semble-t-il, la partie qui apporte le plus de nouveautés, en outre fort utiles aux enseignants. Quatre chapitres, tous de bonne facture suffisent. Olivier Lalieu déroule les expressions de la mémoire qui dès 1945 se sont concrétisées par l'apposition de plaques, par l'érection de monuments, par des manifestations publiques, par des œuvres littéraires ou cinématographiques etc. La voix était accordée aux victimes. Mais la mémoire de la déportation raciale se perdait dans la mémoire de la déportation en générale qui pendant de longues années occulta la particularité de la persécution des Juifs. La confusion perdura et pourtant les chiffres sont là pour mettre en évidence la différence entre les

\footnotetext{
${ }^{9}$ Parmi ses ouvrages : La survie des juifs en France (1940-1944), Paris, CNRS éditions, 2018.

${ }^{10}$ L'article de Monique Heddebaut paru dans notre $\mathrm{n}^{\circ}$ 70, automne 2015-hiver 2016, « Sans armes face à la rafle du 11 septembre 1942, dans la Zone rattachée à Bruxelles », p 119-178, non mentionné, aurait été utile.

${ }^{11}$ Il en est de même dans le nord de la France soumis au commandement militaire de Bruxelles.
} 
déportés politiques et les déportés victimes du racisme : les premiers revinrent dix fois plus nombreux que les seconds qui en outre comptaient des enfants, des vieillards. Et à leur retour, les déportés étaient noyés dans le grand nombre des prisonniers de guerre (950 000 prisonniers). Les Juifs étaient une « minorité dans la minorité » et « des victimes de second rang ». Il y eut bien des associations de déportés mais elles regroupaient trop souvent tous les déportés ${ }^{12}$. Le procès Eichman, à Jérusalem en 1961, éveilla les consciences sur la particularité de la Shoah puis divers événements contribuèrent à faire la différence : la fondation de l'association des Fils et Filles de Déportés de Serge et Beate Klarsfeld (1979), le discours du président Chirac (1995), la Fondation pour la Mémoire de la Shoah (2000) et des œuvres cinématographiques marquèrent les esprits (la série Holocauste et le film Shoah de Claude Lanzman).

«Les voyages de mémoire sur les lieux de la Shoah » sont analysés par Alexandre Bande et Pierre-Jérôme Biscarat, tous deux engagés dans des démarches pédagogiques pour transmettre la mémoire de la Shoah. Ils s'accordent pour souligner l'importance d'une visualisation de la persécution. Il ne s'agit pas toujours de visiter un camp comme celui d'Auschwitz, les élèves peuvent être amenés sur un tout autre lieu de mémoire, comme la Maison d'Izieu où P-J Biscarat exerça de nombreuses années : une démarche dont les retombées s'avèrent très positives pour la transmission de la mémoire de la Shoah. Citons encore parmi bien d'autres lieux: Drancy (et son wagon du souvenir), Beaune-laRolande, Pithiviers, Gurs, Rivesaltes etc ${ }^{13}$. Enfin des souvenirs de déportés et des œuvres littéraires comme celles de l'écrivain Aharon Appelfeld sont aussi autant de lieux de mémoire à grande valeur pédagogique.

Suite à cet article véritable guide pour l'enseignement de la mémoire de la Shoah, Ygal Fijalkow et Jacques Fijalkow interrogent sur les effets que produisent les visites scolaires d'Auschwitz-Birkenau. Constatant que ces visites sont de plus en plus nombreuses, ces deux dernières décennies, les auteurs s'attachent à donner quelques conseils aux enseignants, insistant sur l'indispensable préparation car l'émotion ne doit pas être le but premier voyage. Celui-ci doit éveiller la conscience politique du visiteur, éradiquer ses préjugés sur le racisme et l'antisémitisme. La visite n'est pas touristique mais elle doit faire percevoir des valeurs morales. Et finalement, il revient à chaque enseignant d'ajuster la préparation à son public car « toutes les visites ne se ressemblent pas » (p. 264).

$\mathrm{Au}$ terme de cette partie, Iannis Roder, enseignant d'histoire dans un collège de la banlieue parisienne et attaché au Mémorial de la Shoah, expose «Les défis de l'enseignement de la Shoah ». L'article offre une aide fort utile aux enseignants afin de surmonter les difficultés qu'ils peuvent rencontrer en présentant la persécution raciale. Il insiste sur la nécessité de montrer une histoire positive des Juifs et éviter d'en faire les éternelles victimes d'une fatalité inévitable. L'éducation à la citoyenneté doit contrer cette fatalité. Les témoins peuvent abonder dans ce sens en évoquant leur parcours de déportés mais aussi de résistants. Et surtout, l'auteur conseille d'adapter l'enseignement à son public d'élèves « car chaque âge ne peut être soumis aux mêmes images » (p. 276). Quatrième partie : Questions sensibles.

Cinq questions sensibles sont étudiées, souvent appartenant à l'actualité. Trois d'entre elles ne sont pas apparues récemment : la connaissance des Alliés quant à l'extermination des Juifs (Willy Coutin), les rapports entre le monde arabe et l'Allemagne nazie (Dominique Trimbur) et le négationnisme de 1948 à 2021 (Valérie Igounet) sont des

\footnotetext{
${ }^{12}$ Dès 1955, Lili Leignel petite fille juive déportée de Roubaix à Ravensbrück, à l'âge de 11 ans, témoignait dans le cadre d'une association d'anciens résistants.

${ }^{13}$ Ajoutons pour les Hauts-de-France : la caserne Dossin à Malines ou les parcours de mémoire mis en place dans quelques villes du Nord et du Pas-de-Calais avec le soutien du Mémorial de la Shoah et l'Inspection académique.
} 
questions que ces trois auteurs ont d'ailleurs déjà travaillées et les trois bibliographies citent des ouvrages très récents mais aussi plus anciens des années 1980.

Willy Coutin redit que les Alliés pouvaient être informés sur les tueries, les voies d'information étaient multiples, vers la Grande-Bretagne, les USA et l'URSS. Il rappelle le rôle, vers l'Ouest, de Ian Karski, désormais bien connu, ainsi que les révélations provenant du Bund, du ghetto de Varsovie par Ringelblum ou encore du Joint. Mais la priorité des Alliés était de gagner la guerre et le gouvernement à Moscou restait silencieux.

La présence du grand mufti de Jérusalem dans les couloirs du pouvoir à Berlin est bien connue également mais Dominique Trimbur élabore, fort heureusement, un état de la question et en donne une fine étude historiographique. Puis il évoque quelques paradoxes. Dès la fin du XIX siècle l'Allemagne portait son intérêt sur la Palestine et le MoyenOrient et s'intéressait au sionisme. Hitler considérait les Sémites comme une race inférieure, ce qui n'empêcha pas des nationalistes arabes opposés au sionisme d'adhérer à son idéologie. En revanche des musulmans ont été reconnus Justes parmi les Nations pour avoir aidé des Juifs, surtout en Albanie. Question complexe s'il en est.

Quant à l'histoire du négationnisme, Valérie Igounet rappelle que ce fut une invention française dès le lendemain de la guerre (Bardèche et Rassinier). Puis la Guerre des Six Jours eut pour conséquence la dénonciation de l'impérialisme juif. Enfin vint Faurisson qui dès les années 1980 affirmait que l'histoire de la Shoah était falsifiée. D'autres noms apparaissent : Noam Chomsky, Roques, J-G Cohn-Bendit, Garaudy et le succès du négationnisme auprès de Le Pen. Enfin la seconde Intifada des années 2000 n'a fait qu'amplifier le courant (Dieudonné, Soral).

Avec ces trois questions, une quatrième s'impose: le complotisme associé à l'antisémitisme étudié par le spécialiste de la question : Rudy Reichstadt. C'est une idée ancienne qui refait actuellement surface. Lors des dernières manifestations de rue, en France, la puissance des Rothschild est à nouveau dénoncée, le sionisme est satanique et le droit des Juifs à un État est nié.

Spécialiste du génocide arménien, Yves Ternon interroge : «Peut-on comparer les génocides? " puisque désormais il est reconnu que d'autres génocides ont précédé et suivi celui des Juifs (Hereros, famine en Ukraine, Rwanda, Kmers rouges). La réponse varie selon qu'elle provient du juriste qui tient compte du droit international ou de l'historien qui lui peut dégager trois temps dans le processus génocidaire : la définition d'un groupe supérieur, la mise en place d'un pouvoir totalitaire gangréné par une phobie paranoïaque, l'élaboration de diverses techniques d'assassinats.

Il faut espérer que cet ouvrage parvienne à bénéficier d'un large lectorat notamment parmi les historiens travaillant sur la Shoah mais aussi et surtout parmi les enseignants que la troisième partie concerne particulièrement.

Quelques regrets : l'absence d'index et d'une bibliographie générale qui complèterait les bibliographies qui suivent chaque article ; l'inégalité des articles. Certains se placent à la pointe de la connaissance avec une bibliographie et une historiographie bien développées qui justifient le titre de l'ouvrage, d'autres se limitent à une historiographie datée ou trop générale faisant l'impasse sur des études plus fines.

Danielle Delmaire 